Meta

Journal des traducteurs

Translators' Journal

\title{
L'illustration dictionnairique et les technolectes dans les dictionnaires sémasiologiques
}

\section{Jean Pruvost}

Volume 39, numéro 4, décembre 1994

Hommage à Bernard Quemada : termes et textes

URI : https://id.erudit.org/iderudit/003877ar

DOI : https://doi.org/10.7202/003877ar

Aller au sommaire du numéro

Éditeur(s)

Les Presses de l'Université de Montréal

ISSN

0026-0452 (imprimé)

1492-1421 (numérique)

Découvrir la revue

Citer cet article

Pruvost, J. (1994). L'illustration dictionnairique et les technolectes dans les dictionnaires sémasiologiques. Meta, 39(4), 741-756.

https://doi.org/10.7202/003877ar
Résumé de l'article

L'illustration dictionnairique reste un secteur encore mal connu qui n'a pas suscité beaucoup de recherches. Or la représentation des technolectes au sein d'un dictionnaire passe en partie par les illustrations, qu'il s'agisse des dictionnaires de langue de type sémasiologique ou des dictionnnaires de langue de type onomasiologique, dans lesquels quelques tentatives d'illustration ont pu être repérées. Dans un premier temps, nous présentons donc une genèse correspondant à cette tentation, à partir des dictionnaires fondateurs du XIX ${ }^{\mathrm{e}}$ siècle jusqu'aux récentes réalisations, avec notamment une rapide analyse du Dictionnaire Quillet de la langue française (1948) et du Nouveau Dictionnaire français contemporain (1980), du Dictionnaire manuel illustré des idées par les mots (1898) et du Nouveau Dictionnaire analogique (1980), examinés dans la perspective que nous avons définie. Dans un deuxième temps, en choisissant pour corpus diachronique la "partie langue " $\mathrm{du}$ " Petit Larousse ", suivie à travers douze éditions marquantes, depuis le premier ouvrage de la série à être illustré, le Dictionnaire complet de la langue française (1879), au Petit Larousse illustré de 1994, nous signalons les évolutions significatives, les constantes et la relation dictionnairique établie entre technolectes et illustrations. Enfin, dans un troisième temps, nous proposons une terminologie et quelques analyses. Distinguant entre autres les illustrations simples des illustrations complexes (terminologiques, typologiques, ordonnées, plurielles) et les illustrations monosémiques des illustrations polysémiques, nous procédons à quelques analyses comparatives qui paraissent révélatrices d'évolutions très sensibles. Ne serait-ce que le relevé des termes supportés par les illustrations (810 en 1889, 6280 en 1994). Contribuer par cette première approche à la connaissance des réalisations dictionnairiques, susciter des recherches dans le domaine spécifique des illustrations et des technolectes, tel est ici notre objectif.
Ce document est protégé par la loi sur le droit d'auteur. L’utilisation des services d’Érudit (y compris la reproduction) est assujettie à sa politique d'utilisation que vous pouvez consulter en ligne.

https://apropos.erudit.org/fr/usagers/politique-dutilisation/ 


\title{
L'ILLUSTRATION DICTIONNAIRIQUE ET LES TECHNOLECTES DANS LES DICTIONNAIRES SÉMASIOLOGIQUES
}

\author{
JEAN PRUVOST \\ Université de Cergy-Pontoise, Cergy-Pontoise, France
}

\begin{abstract}
Résumé
L'illustration dictionnairique reste un secteur encore mal connu qui n'a pas suscité beaucoup de recherches. Or la représentation des technolectes au sein d'un dictionnaire passe en partie par les illustrations, qu'il s'agisse des dictionnaires de langue de type sémasiologique ou des dictionnnaires de langue de type onomasiologique, dans lesquels quelques tentatives d'illustration ont pu être repérées.
\end{abstract}

Dans un premier temps, nous présentons donc une genèse correspondant à cette tentation, à partir des dictionnaires fondateurs du XIXe siècle jusqu' aux récentes réalisations, avec notamment une rapide analyse $d u$ Dictionnaire Quillet de la langue française (1948) et du Nouveau Dictionnaire français contemporain (1980), du Dictionnaire manuel illustré des idées par les mots (1898) et du Nouveau Dictionnaire analogique (1980), examinés dans la perspective que nous avons définie.

Dans un deuxième temps, en choisissant pour corpus diachronique la «partie langue» $d u$ «Petit Larousse», suivie à travers douze éditions marquantes, depuis le premier ouvrage de la série à être illustré, le Dictionnaire complet de la langue française (1879), au Petit Larousse illustré de 1994, nous signalons les évolutions significatives, les constantes et la relation dictionnairique établie entre technolectes et illustrations.

Enfin, dans un troisième temps, nous proposons une terminologie et quelques analyses. Distinguant entre autres les illustrations simples des illustrations complexes (terminologiques, typologiques, ordonnées, plurielles) et les illustrations monosémiques des illustrations polysémiques, nous procédons à quelques analyses comparatives qui paraissent révélatrices d'évolutions très sensibles. Ne serait-ce que le relevé des termes supportés par les illustrations (810 en 1889, 6280 en 1994).

Contribuer par cette première approche à la connaissance des réalisations dictionnairiques, susciter des recherches dans le domaine spécifique des illustrations et des technolectes, tel est ici notre objectif.

\begin{abstract}
The "dictionaric" illustration still remains an insufficiently explored field which has not triggered many research studies. Now, the representation of technolects within a dictionary is partly conveyed by the illustration, whether it be semasiologic language dictionaries or onomasiologic language dictionaries, in which some attempts of illustrations have been spotted.

Firstly, we present a genesis corresponding to this illustration attempt, from founding dictionaries of the XIXth century to the recent issues, with, in particular, the quick analysis of the Dictionnaire Quillet de la langue française (1948) and the Nouveau Dictionnaire français contemporain (1980), the Dictionnaire manuel illustré des idées suggérées par les mots (1898) and the Nouveau Dictionnaire analogique (1980), examined in the above mentioned viewpoint.

Secondly, selecting as diachronic corpus the "language part" of the "Petit Larousse", examining successively twelve significant editions, from the first illustrated book, the Dictionnaire complet de la langue francaise (1879), to the Petit Larousse illustré (1994), we point
\end{abstract}


out the significant changes, the constants and the dictionaric relationship between technolects and illustrations.

Thirdly, we propose a terminology and some comparative analyses. Distinguishing simple illustrations and complex illustrations (terminological, typological, serialized, manysided illustrations), monosemic illustrations and polysemic illustrations, we undertake comparative analyses revealing noticeable changes. Only to mention the list of terms connected with the illustrations ( 810 in 1889, 6280 en 1994).

To contribute thanks to this first approach to the knowledge of dictionaric works, to encourage research work in the specific field of illustrations and technolects: such is our aim.

Deux constats sont à l'origine de cette recherche.

Le premier est emprunté à Louis Guilbert évoquant «le mode de signification des termes scientifico-techniques». À propos de ces derniers, il rappelle en effet que «la relation se faisant principalement à partir de la réalité et de la représentation sensible de cette réalité, il s'ensuit que le signe peut prendre une autre forme que le signe linguistique». Et, en l'occurrence, «celle du dessin ou de l'image». «C'est ainsi, ajoute-t-il, que dans les ouvrages techniques le dessin ou la photographie est au nom technique ce qu'est le synonyme ou la définition synonymique au mot commun» 1 .

Le second constat renvoie à Alain Rey signalant à propos des vocabulaires techniques la perpétuelle évolution des structures à dénommer. Et il n'hésite pas à souligner, peutêtre trop abruptement, «l'incapacité des dictionnaires généraux à maîtriser les vocabulaires techno-scientifiques» ${ }^{2}$.

De tels constats trouvent doublement leur source dans la diffusion croissante des sciences et des techniques, et dans le développement parallèle de ce qu'il est convenu d'appeler les «français de spécialité» ou les «français professionnels» ou mieux encore les «technolectes»3. Or un pareil développement n'est pas sans transformer le dictionnaire de langue relevant des classements formels: les technolectes ont en effet nécessairement leur traduction particulière dans les dictionnaires généraux de type sémasiologique. Et l'une de ces traductions passe par les illustrations, celles-ci représentant un système signifiant second qui s'intègre à la définition du signe concerné. Ainsi, les illustrations s'inscriventelles dans une relation dictionnairique particulière qu'il reste à définir.

\section{LE DICTIONNAIRE DE LANGUE ET LA TENTATION DE LILLUSTRATION}

«ll existe des traditions lexicographiques nationales; le dictionnaire de langue est, en France, dépourvu d'illustrations (...). La tradition américaine, elle, ne pratique pas cette dichotomie. L'encyclopédie est profondément mêlée à la langue et l'illustration toujours souhaitée» ${ }^{4}$ remarque presque avec regret Josette Rey-Debove en 1971.

Ce n'est que très récemment en fait que quelques dictionnaristes français ont cependant succombé à cette tentation, la tentation de l'illustration, dans le cadre d'un dictionnaire de langue sémasiologique. Si de fait l'on se réfère exclusivement au dictionnaire de langue, il ne convient pas de retenir au XIX ${ }^{\mathrm{e}}$ siècle le Dictionnaire universel, panthéon littéraire et encyclopédie illustrée de Maurice La Châtre de 1854, en deux volumes, pas plus que l'ouvrage en deux volumes de Bertet Dupiney de Vorepierre intitulé Dictionnaire français illustré et Encyclopédie universelle, publié en 1863, ou même les trois volumes du Dictionnaire français illustré des mots et des choses, publié en 1885, par Larive et Fleury, auteurs alors réputés d'un Cours de Grammaire et langue française. Malgré les intitulés ambigus de ces dictionnaires et les déclarations des auteurs dans les préfaces respectives, ces trois ouvrages restent en effet de caractère nettement encyclopédique.

Il importe néanmoins d'évoquer ces dictionnaires car tous trois sont bien parmi les premiers à être illustrés, et en cette seconde et féconde moitié du XIX ${ }^{\mathrm{e}}$ siècle ils se révèlent 
des pionniers en la matière. Au point que, non sans un certain dépit qui transparaît dans l'ironie du ton, Pierre Larousse, procédant dans la Préface du Grand Dictionnaire Universel à l'analyse du travail de Dupiney de Vorepierre, signale que si «la partie lexicologique du livre de M. Dupiney fait assez pauvre figure (...) en revanche la partie encyclopédique est assez riche; elle ne se contente même pas d'être riche, elle frappe l'œil par le luxe qu'elle étale: des gra-vures! des gravures! » Et Pierre Larousse d'ajouter perfidement: «Était-il bien nécessaire, dira-t-on, de nous offrir l'image d'un âne, à l'article âne. On pouvait peut-être s'en passer, répondrons-nous ; mais après tout, si cela n'apprend rien, cela peut amuser les enfants de voir au milieu de ce texte ces oreilles si connues». Ironie du sort, l'âne fera son entrée dès 1906 dans le Petit Larousse Illustré pour ne plus jamais en sortir. Il accède même à la couleur dans l'édition de 1994...

Il faut donc attendre le XXe siècle avec notamment, en 1948 , le Dictionnaire Quillet de la langue française en trois volumes, rédigé sous la direction de Raoul Mortier, pour qu'apparaissent des illustrations. Ainsi, pour cet ouvrage dont l'épigraphe est sans ambiguïté, «l'art d'écrire et de bien rédiger», il faut dénombrer 74 planches et illustrations qui accompagnent les articles. Et il s'agit bien en effet d'accompagnement car ces planches et illustrations sont données, selon la formule de Raoul Mortier dans l'Avant-Propos, «à leur ordre alphabétique» (p. VIII), afin de pouvoir être consultées parallèlement à la lecture de l'article. Parmi ces 74 planches et illustrations, citons, entre autres, la planche consacrée à l'aéronautique, à la boucherie, à la charpente, aux chaussures ou aux lignes, ainsi que les deux planches dévolues aux machines agricoles, à la botanique, aux maisons, ou bien encore les trois planches réservées aux costumes, aux instruments de musique ou à la navigation.

Pour de tels articles, Raoul Mortier précise dans l'Avant-Propos qu'il convient de «concourir d'une façon visuelle à l'intelligence exacte du sens des mots particuliers dans un ensemble (figures d'ensemble ou mots imprimés sur la fígure même)» (p. IX). À vrai dire, en se limitant à une radiographie du seul premier volume, qui contient 36 planches, ce ne sont pas moins de 1850 termes qui s'y répartissent, avec donc en moyenne environ 50 termes par planche, «cheval» en accueillant par exemple presque 100 , «chaussures» 69 et «arcs» 35. De fait, si les illustrations sont ici limitées au dessin représentatif des choses nommées, prises dans le détail ou le plus souvent insérées dans leur contexte, le souci d'énumération lexicale apparait clairement. Tous les termes présentés ne sont d'ailleurs pas nécessairement repris dans la nomenclature, ils participent alors de l'ensemble présenté dans une perspective de relative exhaustivité. Ainsi en est-il du «diairi» dans la planche consacrée aux costumes ou du «balmoral» et du «coupe-lacet» dans celle réservée aux chaussures.

S'il importe de souligner ici, au sein d'un dictionnaire de langue, la présence d'un essai intéressant d'illustration pour des séries de mots rattachés à des ensembles de type encyclopédique, force est de reconnaître qu'au-delà de l'unique phrase d'explication formulée par Raoul Mortier dans l'Avant-Propos, il n'y a pas là en vérité de stratégie nettement élaborée. La tentation de l'illustration s'y révèle à l'état premier, sans que le dictionnariste n'en tire réellement argumentation: faute d'une réflexion riche, l'illustration reste discrètement très marginale.

C'est ensuite, en 1980, au Nouveau Dictionnaire français contemporain, le N.D.F.C., que revient une nouvelle tentative, avec 1062 illustrations annoncées en regard des 33000 mots de la nomenclature. Cet essai reste d'autant plus intéressant qu'il s'inscrit dans la dynamique de son prédécesseur, le Dictionnaire français contemporain, dictionnaire de langue unanimement apprécié pour son caractère novateur, à la fois expérimental sur le plan lexicographique et courageux sur le plan dictionnairique. Sous la direction de Jean Dubois, avec René Lagane et une nouvelle équipe de rédaction, le N.D.F.C., «en 
gardant les mêmes principes de description de la langue et les mêmes objectifs» que le D.F.C., offre une nomenclature augmentée de 3200 termes nouveaux constituant «le lexique usuel du français contemporain à partir duquel se développent les terminologies scientifiques et techniques». Et dans l'Avant-Propos il est pour le moins intéressant de constater que les auteurs regroupent dans un même paragraphe intitulé Définitions et illustrations les remarques y correspondant, marquant bien ainsi la complémentarité possible des deux codes définitoires. Jean Dubois y évoque alors clairement l'intérêt des illustrations pour les termes techniques. Au-delà en effet du travail de définition, traduction de tous les traits sémantiques définissant le mot dans une structure donnée, «le problème demeure pour les termes techniques qui dénomment des appareils, des plantes, des animaux, etc., la définition étant une simple description de la chose elle-même ou de son utilisation». Est alors rendu hommage à l'image et plus particulièrement au dessin «qui retient les traits distinctifs de l'objet: on les trouvera là où la nécessité s'est fait sentir de confronter les mots avec les objets qu'ils dénomment».

Par ailleurs, comme nous l'avons déjà souligné pour le Dictionnaire Quillet de la langue française, l'illustration représente aussi une échappée du dictionnaire vers l'extensivité, le technolecte qui n'a pu être accueilli dans la nomenclature retrouve une dimension dans son environnement naturel, projeté graphiquement dans le dictionnaire: «les illustrations comportent aussi des mots désignant des parties d'un objet, d'un organisme, les détails d'un fonctionnement» et «ces termes qui appartiennent à des nomenclatures étendues sont suffisamment définis par l'image pour n'avoir pas besoin d'être repris dans le corps du dictionnaire». Si l'on se fie en effet aux intentions et à l'argumentaire, il ne fait aucun doute que l'illustration trouvait ici une place définie, dans le cadre d'une stratégie dictionnairique qui ne la réduisait pas à un «luxe». Au reste, la conclusion du paragraphe ne manquait pas de panache: «Le N.D.F.C. est donc illustré pour jouer pleinement son rôle de dictionnaire. Les illustrations, par les termes qu'elles contiennent, sont une première étape dans la connaissance du français technique et scientifique». Il semblait bien qu'il y avait là une ouverture et une recherche dont les lendemains seraient fructueux. Hélas, lorsqu'en 1987 est lancé le Dictionnaire du français au collège, successeur du N.D.F.C., il n'y a de nouveau plus de place pour les illustrations dans le dictionnaire de langue alphabétique.

Certes, à cet abandon rapide correspond une explication immédiate et simple avancée par les Éditions Larousse, il fallait trouver de la place aux 2000 nouveaux termes ajoutés et, les illustrations ayant pour défaut premier d'être particulièrement grourmandes en espace typographique, leur disparition à permis cet ajout. Mais si celles-ci avaient tenu leurs promesses et séduit le public, il est assuré qu'elles n'auraient pas subi un sort aussi radical. Ainsi, une radiographie de la partie du N.D.F.C. réservée à la lettre $\mathrm{A}$ se révèle très éclairante sur la manière dont ce dictionnaire a été mis en œuvre quant aux illustrations. En fait, si la préface ne manque pas d'intérêt et paraît dans ce domaine prometteuse, le programme d'illustration est inexistant: sur les 87 illustrations choisies pour les mots de la lettre A, 39 sont issues du Petit Larousse 5 de 1959, 20 du Nouveau Petit Larousse ${ }^{6}$ de 1968, une du Petit Larousse illustré de 1973 (ananas), et seules trois illustrations se retrouvent dans celui de 1981 (aloès, arum, aulne). Quant à l'illustration représentant une chèvre angora, elle remonte même à l'édition de 1952 .

Nous n'avons pas réussi à identifier les illustrations restantes, mais il est probable qu'elles sont également issues d'un imagier déjà existant et l'on peut conclure sans état d'âme qu'il n'y a pas eu de programme spécifique d'illustration pour le N.D.F.C., en dépit de l'énoncé théorique pertinent de l'Avant-Propos. Ajoutons, s'il en était besoin, que l'agami et l'agouti déjà présents dans le Dictionnaire complet de la langue française publié en 1879, le premier dictionnaire Larousse à être illustré et le véritable ancêtre du Petit Larousse, ne sont sans doute pas des animaux essentiels dans le vocabulaire usuel. Sans 
oublier qu'ils doivent sans doute leur insertion dans le dictionnaire de 1879 au fait qu'il sont représentés dès 1854 dans le dictionnaire de La Châtre déjà cité. Quant à l'aï, ou si l'on préfère le paresseux, en dehors des mots croisés où il a une place d'honneur, il ne mérite sans doute pas que l'on s'y attarde. D'autant plus que ce tardigrade d'ordinaire suspendu se retrouve ici, par une erreur révélatrice du peu de soin apporté à l'illustration, disposé en sens inverse, le dos arqué vers la cime de l'arbre. Enfin, il est aisé de le constater à travers les articles choisis pour être illustrés, le vocabulaire technique n'y est guère représenté, en tout cas l'effort discret des auteurs du Dictionnaire Quillet de la langue française était infiniment plus représentatif à cet égard. Pour les 87 illustrations correspondant à la lettre $\mathrm{A}$ dans le N.D.F.C., nous ne dénombrons que 171 termes rattachés à l'ensemble des illustrations, au lieu des 1850 repérés dans le Dictionnaire Quillet de la langue française. L'on comprend donc aisément que, dès 1987 , c'est sans regret que les illustrations disparaissent au profit des 2000 mots supplémentaires du Dictionnaire du français au collège. On peut regretter que cette recherche ait tourné court, mais le point de vue de Jean Dubois, à défaut d'avoir connu une application dictionnairique, n'en reste pas moins pertinent et incitateur.

Si l'on se tourne maintenant vers les dictionnaires de langue de type onomasiologique, il reste actuellement un dictionnaire pour lequel la tentation de l'illustration semble avoir abouti et paraît être pérennisé de réédition en réédition. Il s'agit du Nouveau dictionnaire analogique rédigé sous la direction de Georges Niobey, dictionnaire qui fait suite à celui de P. Boissière, promu et édité en 1862 par Pierre Larousse, et à celui de C. Maquet publié en 1862 par la même Librairie Larousse. Ni le tout premier du genre, le Dictionnaire analogique de la langue française, celui de Boissière, ni le Nouveau dictionnaire analogique de C. Maquet n'avaient fait appel à l'illustration.

Parmi ces dictionnaires de langue particuliers que sont les dictionnaires analogiques, seul en fait Paul Rouaix avec son Dictionnaire manuel illustré des idées suggérées par les mots avait réservé, dès la première édition en 1898 , quelques pages aux illustrations. Áinsi «16 planches de figures hors texte» sont offertes en toute fin d'ouvrage, en l'occurrence 16 pages constituées de 29 figures se suivant dans 1'ordre alphabétique. Alambic, ancre, armure, bicyclette, blason, canon, cerveau, charrue, cœur, colonne, corps, crâne, dents, digestion, fleur, harnachement, horloge (mouvement d'), lampe, insecte, locomotive, muscles, navire, œil, oiseau et œuf, ornements principaux, poisson, squelette d'homme, voilure, voiture, tels sont les thèmes retenus rassemblant sous ces intitulés 660 termes, ce qui représente en moyenne un peu plus de 20 termes par figure. Contrairement au dictionnaire de type sémasiologique, tous les termes présentés dans ces planches semblent retrouver leur place sous les mots-centres du corps de l'ouvrage. Il est vrai que, par essence, le dictionnaire analogique, dépourvu de définitions (à l'exception du dictionnaire fondateur de Boissière) est généralement riche en technolectes. Avec 1718 mots-centres et 91000 mots-analogues, le dictionnaire de Paul Rouaix, bien que l'un des moins riches en mots analogues (l'ouvrage de C. Maquet en compte 138 000), est forcément plus accueillant que les dictionnaires sémasiologiques destinés à un public comparable. Cela étant, si les illustrations proposées sont ici relativement soignées et précises, elles ne seront pas remises à jour, et il va sans dire que les thèmes retenus en synchronie portant nécessairement la marque de leur époque, très rapidement, il ne sera plus possible de conserver telles quelles des planches datées. Or le dictionnaire analogique, de par le fait même que n'y sont pas données les définitions des mots enregistrés et classés, réussit plus que son homologue sémasiologique à perdurer, traversant parfois plusieurs états de langue, sans qu'au premier regard l'on repère les éléments manquants.

À cet égard, le dictionnaire de Paul Rouaix représente bien un champion de l'épaisseur synchronique puisqu'en 1981 il était encore vendu tel quel, sans qu'une colonne n'en ait 
été transformée, mais bien entendu, depuis plus d'un demi-siècle, les illustrations avaient disparu. Si l'alambic, le harnachement, la locomotive de 1898, la voilure d'un navire, la voiture à cheval, ainsi que les différentes pièces désignées dans ces planches pouvaient discrètement rester nichés en tant que mots dans les colonnes du mot-centre correspondant, au nom d'une certaine panchronie, le thème illustré devenait dictionnairiquement par trop désuet, la suppression des illustrations s'est imposée assez vite. Sans doute aussi, faute d'une stratégie clairement établie: la préface de Paul Rouaix, alors professeur au Lycée Henri IV, ne faisait en effet aucune allusion aux illustrations. Il semble bien que l'illustration était, en la circonstance, davantage affaire d'éditeur que d'auteur, le Dictionnaire des idées suggérées par les mots s'intégrant en effet dans une collection intitulée La Bibliothèque des Dictionnaires manuels illustrés.

Aussi lorsqu'en 1980 réapparaissent les illustrations dans le Nouveau dictionnaire analogique de Georges Niobey, illustrations en noir et blanc disséminées dans le corps de l'ouvrage, sous la forme de planches et de figures, il ne s'agit pas, à dire vrai, d'une filiation naturelle dans le cadre des dictionnaires analogiques, mais plutôt d'une innovation qu'il convient de rattacher aux propos de Jean Dubois. Au reste, ce dernier dictionnaire et le Nouveau dictionnaire analogique ont un dénominateur commun, tous deux ont bénéficié de la compétence de René Lagane qui a fait partie de chacune des deux équipes de rédaction. C'est cependant avec une grande discrétion qu'est prise cette initiative. Les planches et les figures ne sont ni annoncées en couverture, ni évoquées en sous-titre, ni même répertoriées.

Loin d'en tirer avantage, les auteurs passent en fait presque sous silence leur existence. Et dans l'Avant-Propos, seule une phrase lapidaire du chapitre consacré à l'Historique des dictionnaires analogiques Larousse y fait référence: «Nous avons également ajouté un certain nombre d'illustrations qui suppléent au manque de définitions» déclare sans plus d'explications Georges Niobey.

Et pourtant si l'on feuillette l'ouvrage, ce ne sont pas moins de 38 thèmes qui $y$ sont illustrés avec 28 planches et 10 figures?. À mieux y regarder encore, avec un total de 41 pages concernées sur les 780 offertes hors index, ce sont environ $5 \%$ de l'ouvrage qui sont ainsi réservés aux illustrations. Des pages qui se révèlent en réalité porteuses d'un vocabulaire technique et scientifique non négligeable puisque, en ne considérant que la lettre $\mathrm{A}$ et les planches s'y référant, ce sont déjà presque 250 termes qui s'y retrouvent représentés. Et si c'est l'ensemble de l'ouvrage que nous radiographions, il faut alors dénombrer 2200 termes ainsi explicitement désignés au sein des illustrations, ce qui est loin d'être négligeable, et correspond en moyenne à 58 signes par planches, mis en relation directe avec leur référent. Or, il est clair que les thèmes ici illustrés, selon des processus d'illustration variés et souvent originaux, relèvent tous ou presque de vocabulaires spécifiques auxquels l'illustration offre entre autres un environnement visuel ainsi qu'une mise en situation. Il s'agit là en fait pour les technolectes d'une représentation dictionnairique particulière qu'il n'est pas possible d'ignorer.

Ainsi, à ce que nous appelons ici la tentation de l'illustration au sein des dictionnaires de langue, correspondent bien des initiatives diverses, aussi bien dans les classements formels que dans les classements sémantiques. Mais de fait, si pour un «Petit Larousse», de facture explicitement encyclopédique, il reste assurément de bon ton d'annoncer systématiquement le nombre d'illustrations offertes aux lecteurs, inversement pour un dictionnaire de langue, il semble bien que valoriser l'illustration paraît encore incongru au regard de notre tradition bien ancrée de l'austère dictionnaire de langue, ouvrage monolingue servi par un seul code, celui de la langue décrite. Cependant, grâce à la distinction fondamentale établie par B. Quemada entre la lexicographie d'ordre pleinement scientifique et qui n'a pas explicitement pour finalité l'élaboration d'un dictionnaire destiné au grand public, et la dictionnairique ayant pour objet et finalité «le genre dictionnairique» 
en tant qu'instrument de communication, ensemble documentaire systématique et produit technico-commercial, cette réserve vi-à-vis des illustrations disparaît utilement, notamment lorsqu'il s'agit des technolectes. L'illustration peut alors devenir complémentaire de l'article et répondre à une stratégie cohérente.

Au moment même où l'informatique offre à la dictionnairique de nouveaux espaces et de nouvelles dimensions, il convient d'offrir à ce système signifiant second toute sa place, en essayant de l'intégrer dans une réflexion d'ensemble. Car il s'agit en fait d'aboutir à une programmation efficace des différents processus d'illustration qui puisse se révéler à la fois homogène, rigoureuse et représentative pour le consultant. Et décrire et analyser ce qui a déjà été tenté dans ce domaine représente sans doute la première étape d'un tel programme dictionnairique.

\section{TECHNOLECTES ET ILLUSTRATIONS DANS}

\section{LA «PARTIE LANGUE» DU «PETIT LAROUSSE» DEPUIS 1879}

Si manifestement les tentatives d'illustration sont restées très discrètes dans les dictionnaires de langue, elles demeurent la marque patente de la «partie langue» 8 des dictionnaires encyclopédiques. Et parmi ceux-ci nous retiendrons notamment le «Petit Larousse» à travers ses diférentes métamorphoses et ses différents titres.

Ce dernier correspond en effet à une histoire dictionnairique étalée sur plus d'un siècle et englobant tout le $\mathrm{XX}^{\mathrm{e}}$ siècle. De fait, à la suite du Nouveau Dictionnaire de la langue française de Pierre Larousse, publié en 1856, dictionnaire non illustré, le premier à bénéficier d'illustrations est le Dictionnaire complet de la langue française, publié en 1879. Selon les propres termes de la préface (p. 13), «cette innovation est capitale», elle offre à la consultation «environ 1500 figures, complétant et précisant le sens des mots définis, en donnant une idée de la forme des objets et des êtres qu'ils représentent». Ainsi, d'emblée, en sélectionnant des objets, se pose déjà inévitablement le problème de la représentation dictionnairique des technolectes. Et d'édition en édition, de refonte en refonte, selon un code qui s'élabore au fur et à mesure, avec des procédés matériels de plus en plus variés, les illustrations en constante évolution sémiotique se prêtent à une étude à la fois analytique et comparative.

Aussi est-il possible de choisir pour corpus d'une étude diachronique l'ensemble des différentes éditions du «Petit Larousse», c'est-à-dire depuis l'édition de 1879, la première à être illustrée, jusqu'à celle de 1994, où «les dessins, les photographies, les cartes et les schémas éclairent les mots, prolongent les définitions» et «les planches mettent en relation les éléments dispersés par l'ordre alphabétique».

Pour être plus précis, ce sont successivement les douze éditions marquantes de l'histoire spécifique du «Petit Larousse» qui ont été ici examinées, de l'édition de 1879 à celle de 1994, afin de saisir, dans notre troisième partie, les évolutions significatives, les constantes et la relation dictionnairique établie entre technolectes et illustrations. Nous nous sommes cependant limité à l'examen de la lettre A qui représente en gros $10 \%$ des illustrations de la «partie langue» du dictionnaire. Il n'était guère possible en effet, et surtout guère rentable, de suivre une évolution répartie sur toutes les lettres de l'alphabet et donc sur plusieurs milliers d'illustrations, la seule édition de 1994 comportant par exemple 3600 illustrations.

Avant toute analyse, il importe de décrire rapidement ce que chacune de ces douze éditions successives présente de particulier, examinée dans la perspective commune des technolectes et des illustrations.

L'édition de 1879, fondatrice du genre avec 1500 figures destinées à illustrer «la langue française», publiée vingt-trois ans après l'ouvrage initial de Pierre Larousse, est d'emblée présentée sous un jour sémiotique. Il s'agit pour le moins, si l'on en croit la 
préface de ce Dictionnaire complet de la langue française, d'un «répertoire éminemment utile, écrit en deux langues, se prêtant un mutuel appui : la langue maternelle, restreinte à notre nationalité; la langue du dessin, commune à tous les peuples, Universelle, en un mot».

Y est reprise par ailleurs la préface de l'ouvrage précédent, le Nouveau Dictionnaire de la langue française de 1856, préface rédigée par Pierre Larousse et dans laquelle il expose les limites de la définition pour certains mots. Ce qui laissse présager ainsi indirectement l'adjonction des illustrations: «Notre langue comporte un grand nombre de mots pour lesquels une définition, quelque étendue qu'elle soit, reste toujours obscure. La curiosité du lecteur n'est qu'éveillée, son esprit n'est point satisfait. Il nous suffira de citer les mots aérolithe, alchimie, aristarque (un), fossile, galimatias, patelin, phrénologie, tramontane, vaccin, etc. Ici une notice encyclopédique, nous voulons dire étymologique, scientifique, historique ou littéraire, était indispensable (...). Plus de quatre cents mots sont suivis de ce précieux appendice» (p.10). Ces notices encyclopédiques, qu'elles soient étymologiques ou scientifiques, dans la mesure où elles sont dans l'esprit de Pierre Larousse destinées à éclairer les définitions de certains mots, des mots techniques entre autres, préfiguraient déjà de fait l'adjonction d'illustrations.

Pour l'édition de 1889 est adopté le titre plus explicite de Dictionnaire complet illustré, avec cette fois-ci 2000 figures annoncées, l'ensemble d'ouvrage étant sous la direction de Claude Augé, très attaché au «développement méthodique du principe fécond de 1'illustration». La «partie langue», celle qui nous préoccupe et qui se trouve située avant les célèbres «pages roses», accueille dès cette édition davantage d'illustrations d'ordre technique, notamment sous la forme de planches, avec par exemple la «locomotive» et le «cuirassé d'escadre La Dévastation». Une «note des éditeurs» (p. 8) insérée sous la présentation des «Tableaux encyclopédiques» est révélatrice de l'importance des illustrations pour Claude Augé : «Nous tenons à rappeler ici que, les premiers en France, nous avons ouvert la voie féconde de l'illustration des dictionnaires manuels. Comme il convient de rendre à chacun ce qui lui est dû, disons aussi que l'idée première de quelquesuns des Tableaux ci-dessus, ceux qui sont marqués d'un astérisque, nous vient de deux ouvrages de langue anglaise, le Dictionnaire de Webster et le Vocabulaire symbolique de Ragonot. Tous les autres sont entièrement originaux : ils ont été composés expressément pour le Dictionnaire Larousse.»

À l'édition de 1906 correspond un format plus volumineux et le titre qui fera florès de Petit Larousse illustré. Sont alors annoncées 5800 gravures distribuées dans le texte et 130 tableaux dont quatre en couleurs, «d'une facture très fine» et «essentiellement documentaire: la fantaisie n'y a aucune part». Il s'agit, déclarent les Éditeurs, d'autant de «synthèses en image dont la portée éducative et la valeur suggestive sont incontestables». Pour la première fois, y sont également cités avec leurs diplômes, et donc leur spécialité, les collaborateurs choisis «pour assurer la plus grande précision des définitions techniques». Paul Augé y apparaît «licencié ès sciences physiques et chimiques» aux côtés de Gaston Boucheny, «licencié ès sciences physiques et mathématiques». Enfin, il faut signaler l'apparition de la légende pour chaque illustration et l'augmentation très sensible des figures d'ordre technique. Ainsi, témoignant parallèlement de la montée vers la Première Guerre mondiale, phénomène sensible tout au long de l'ouvrage, l'illustration du canon de $75 \mathrm{~mm}$, en rassemblant pas moins de 29 termes techniques, est à cet égard très symbolique. Y font irruption également des planches consacrées aux sciences, par exemple celle des microbes observés au microscope.

L'édition de 1935, avec Paul Augé, «licencié ès sciences physiques et chimiques», comme Secrétaire général de la rédaction, a pour titre celui, adopté en réalité dès 1924, de Nouveau Petit Larousse illustré. Doté de 6200 gravures, 220 planches et tableaux, cette édition fait l'objet d'une «élimination des détails oiseux» et d'un «triage judicieux des 
mots» propre à donner «des développements plus considérables aux articles encyclopédiques».

L'édition de 1948 est entièrement refondue, avec 6400 gravures, 220 planches et tableaux, dont des «photographies en similigravure». Le vocabulaire y est enrichi de près de 12000 acceptions nouvelles et «on trouve les termes relatant les inventions et découvertes récentes, celles du radar, de la fission de l'uranium, par exemple», le tout «grâce aux observations conjuguées et coordonnées de cette nombreuse équipe de linguistes éminents, de fins lettrés, de spécialistes qualifiés et d'habiles techniciens».

L'édition de 1952 est celle du centenaire de la Librairie Larousse avec 4130 illustrations en noir, 44 hors-texte en couleurs et en noir, rédigée sous la direction de Claude et Paul Augé. Les principaux collaborateurs y sont listés séparément ( $p$. III), selon qu'ils représentent les «Lettres: grammaire, littérature, histoire, géographie, beaux-arts, etc.» (notons au passage la présence de R. Bailly, de Claude Dubois, de Georges Niobey et de Michel de Toro) ou bien les «Sciences: mathématiques, sciences physiques et naturelles, chimie, etc.». Si «la partie consacrée à la langue française» est légèrement renouvelée, c'est de fait surtout «la partie Arts, lettres, sciences» qui a subi dans cette édition un complet remaniement.

Pour l'édition de 1959 est adopté le titre simplifié de Petit Larousse, avec, de nouveau, un format augmenté qui «ménage une place accrue à l'illustration», en la circonstance 5130 illustrations en noir et 48 pages en couleurs. En vérité, il faut noter la présence d'une marge, presque de la taille d'une colonne, une marge qui est particulièrement fournie en dessins et schémas, souvent d'ordre technique, révélateurs de l'époque, tels que le servo-frein, l'amortisseur, l'alternateur. «Non seulement aucun cliché de l'ancienne illustration ne subsiste, mais tout sujet pouvant paraître vieilli ou superflu a été supprimé et des ensembles ont été consacrés aux techniques modernes. Les dessins deviennent plus explicatifs que descriptifs, la photographie, plus nombreuse elle aussi, les remplaçant quand c'est nécessaire.» Par ailleurs, l'illustration «qui se veut intégralement utile» est améliorée grâce à l'utilisation du procédé offset pour l'impression. Enfin, sont acceptés dans cette édition «certains termes étrangers employés maintenant dans les diverses techniques et même dans le vocabulaire courant. En revanche, de nombreux termes désuets ont été écartés, en particulier ceux qui se rapportaient à des techniques périmées».

L'édition de 1968 est présentée sous un nouveau titre, le Nouveau Petit Larousse, et les articles s'étalent sur trois colonnes à la faveur d'une légère augmentation du format, permettant «d'accroître la surface réservée à l'illustration», 5535 illustrations, 56 pages en couleurs, sans oublier l'ajout de plusieurs planches supplémentaires, en noir, «consacrées aux arts et à la technologie». La «partie langue» est soumise à «une réfection systématique», puisque «les transformations considérables qu'ont connues depuis quelques années tant les sciences fondamentales que les sciences appliquées et les sciences humaines ont provoqué un renouvellement du vocabulaire».

Pour l'édition de 1973 est adopté le titre désormais acquis jusqu'à l'édition de 1994 de Petit Larousse illustré. À cette édition, riche de 5535 illustrations en noir et de 56 pages en couleurs, ne correspond pas à vrai dire une notable évolution dans le corps de l'ouvrage.

L'édition de 1981 est par contre l'occasion d'une refonte importante «tant du point de vue du contenu que de celui de la présentation». Les 4180 illustrations, les 54 pages en couleurs y sont conçues en effet comme relevant d'une illustration «essentiellement didactique et documentaire», une place importante étant réservée aux dessins et aux photographies «choisies en fonction de 1'information dont elles sont porteuses». Par ailleurs, résultat de la collaboration de linguistes et de spécialistes des différents secteurs de la connaissance, comme le rappellent les Éditeurs, ce dictionnaire fait «une large place, aux côtés des mots de la langue commune, aux termes et sens, nombreux et variés des grands 
domaines de la culture contemporaine et à ceux qui relevant de disciplines plus spécialisées, ont débordé dans leur emploi leur domaine d'origine». Il s'agit de «rendre compte avec fidélité de l'évolution de la langue et du monde». Il est au reste intéresssant de constater que bien des photographies apparues dans les éditions précédentes, notamment depuis 1959, sont remplacées par un dessin. Enfin, de plus en plus, il est fait appel aux schémas, aux vues en coupe, aux écorchés, qu'il s'agisse de l'aéroglisseur, du char français AMX, du bathyscaphe, ou du radiateur à accumulateur de chaleur.

L'édition de 1989 est également l'objet d'une refonte et comporte 3600 photos et dessins. Elle se définit dans la préface comme une «révolution sans bruit ni fureur, intégrant les plus récents progrès de la lexicographie», préservant par ailleurs la «pertinence et variété de l'illustration» avec près de 1850 dessins, plus de 1750 photographies, près de 250 cartes et documents divers». Enfin, «fidèle à sa tradition encyclopédique, le Petit Larousse 1989 a intégré, au premier chef, de nombreux termes spécifiques des sciences et des techniques d'aujourd'hui, notamment en médecine, informatique, biologie, sciences de l'ingénieur». Sans doute révélatrice d'un univers plus technicisé et d'un public plus averti à cet égard, sont annoncés de plus en plus souvent dans les légendes les objectifs poursuivis à travers l'illustration offerte. Le métalangage ainsi utilisé s'adresse désormais à des lecteurs soucieux de comprendre au-delà des apparences. Ainsi, il n'y a plus de «lichen», de «fossile», de «hiéroglyphes», mais un «exemple de lichen», un «exemple de fossile», etc. De même, les «schémas de fonctionnement» (de l'imagerie médicale, du laminoir, etc.) font pendant aux «schémas d'élaboration» (de l'acier, de l'aluminium, etc.) et aux «schémas de principe» (des microscopes, du tokamak, etc.). Et les «écorchés» (par exemple «l'écorché d'une rame MF 77 utilisée par la R.A.T.P. à Paris» qui illustre l'article «métro») s'y multiplient, annoncés en légende. Mais aussi non annoncés: ainsi, pour dix écorchés que nous avons comptés explicitement présentés dans la légende, ce sont presque 70 écorchés non légendés qui ont été dénombrés dans la «partie langue», de la «ruche à cadres mobiles» à la «perceuse électrique» en passant par la «lecture d'un disque numérisé».

Pour conclure ce rapide parcours diachronique, il faut citer l'édition de 1994 qui ne diffère pas fondamentalement de celle de 1989, mais qui se démarque par le fait qu'il s'agit de la première édition où toutes les illustrations sont en couleur, ce qui n'est pas sans importance pour la précision de l'information et une meilleure lecture de l'illustration ainsi plus facilement codée. Avec 3600 dessins, schémas et photographies qui, comme le rappellent les auteurs, «éclairent les mots, prolongent les définitions» tout autant que les planches «mettent en relation les éléments dispersés par l'ordre alphabétique».

\section{UNE TERMINOLOGIE ET QUELQUES ANALYSES}

Évoquer les illustrations, au sein d'une communication de type dictionnairique, c'est évidemment faire appel à un autre code signifiant, à la fois étranger et complémentaire par rapport au code linguistique. Et dans la dictionnairique qui associe ces deux codes, si le champ linguistique de la définition est relativement bien jalonné, connu et reconnu, le système sémiotique de l'illustration reste presque entièrement à cerner. Polymorphe et évolutif, le système illustratif résulte en fait de pratiques ne relevant pas d'un code établi explicitement. Cependant les illustrations s'inscrivent dans le même souci d'économie que celui qui préside à la langue et à la définition de ses constituants. Et dans le cadre dictionnairique encore mal repéré qui associe les définitions et les illustrations se révèle tout particulièrement, selon la formule de B. Quemada dans la «Postface» des Dictionnaires du français moderne (p. 563), le «métier, talent ou technique (parfois l'un et l'autre) qui sut souvent prêter aux auteurs l'intelligence pragmatique des problèmes encore informulés». Aussi en sommes-nous encore au stade des premières approches descriptives et nous ne pourrons ici présenter que quelques pistes de recherche possibles. 


\section{Approche terminologique}

Les premières analyses comparatives des douze éditions se succédant impliquent nécessairement un travail de typologie et la mise en place d'une terminologie. Ainsi, il faut distinguer l'illustration d'un article des figures qui peuvent composer cette illustration. Un article peut n'avoir qu'une figure d'illustration auquel cas nous dirons qu'il est monofigural, il peut également comporter plusieurs figures, et nous l'appellerons alors polyfigural (bifigural, trifigural, etc.).

Par ailleurs, l'illustration polyfigurale peut être monosémique, c'est-à-dire composée de figures différentes pour illustrer un même sens, ou polysémique, c'est-à-dire composée de figures différentes correspondant à des sens différents du mot. Enfin, qu'elle soit légendée ou non, elle peut simplement se rattacher à l'article en offrant un seul signifiant visuel et il s'agit alors d'une illustration simple, ou bien au contraire constituer un relais d'informations et nous l'intitulerons dans ce cas illustration complexe.

L'illustration complexe est diverse. Elle peut avoir pour finalité la présentation d'un microsystème dénominatif, différentes parties de l'objet étant désignées et chaque mot ainsi rajouté ayant une fonction dénotative référentielle, cette illustration ou cette figure est alors terminologique (exemple: l'alambic avec la désignation de ses différentes composantes). L'illustration complexe peut aussi être représentée par une illustration typologique, c'est-à-dire qu'y sont représentés plusieurs types du même référent virtuel décrit, ce qui à la fois neutralise en partie les traits individuels et donne un échantillon de la totalité du possible (exemple: différents types d'abat-jour). Nous distinguons également parmi les illustrations complexes l'illustration plurielle qui présente un même ensemble sous des points de vue différents (exemple : l'ascenseur, 1-la cabine, 2-le moteur, 3-la cage d'escalier). Enfin, nous appellerons illustration ordonnée toute illustration dont les figures répondent à la mise en valeur d'un processus, souvent organisé de manière chronologique ou logique (exemple : le schéma d'élaboration de l'acier, le cycle de l'azote). L'illustration complexe reste bien entendu le lieu de possibles superpositions, une illustration peut par exemple être plurielle et ordonnée, ainsi en est-il du tuyau acoustique dont, en 1906, l'illustration trifigurale est à la fois ordonné selon une logique de montage et selon ses différentes parties (le tuyau, l'embout et le cornet).

Examiner les illustrations successives de l'article «ancre» nous permettra de mieux concrétiser cette terminologie. Dans l'édition de 1878, cet article est en effet tout d'abord accompagné d'une illustration monosémique monofigurale: le simple dessin d'une ancre marine. Dès 1906 cependant, cette illustration est légendée et devient terminologique, quatre parties y sont désignées: l'organeau, le jas, la tige, le bras. Puis en 1959, l'illustration devient polysémique. En effet, trois types d'ancre sont représentés, l'ancre marine, l'ancre de construction et l'ancre d'horlogerie, et, si l'on veut être plus précis, l'illustration est trifigurale pour l'ancre marine, avec l'ancre Martin, l'ancre à jas, et l'ancre Halle, cet ensemble étant donc typologique, monofigurale pour l'ancre d'horlogerie de même que pour l'ancre de construction. Et l'illustration de l'article redevient monosémique en 1968 puisque, effectivement, ne reste plus alors que l'ancre de marine, toujours trifigurale et typologique.

Enfin, s'il fallait aller plus loin, nous reprendrions une distinction faite par $\mathbf{R}$. Barthes, lorqu'il analyse les planches de l'Encyclopédie dans les Nouveaux essais critiques, et qu'il y établit la différence entre la figure paradigmatique qui représente l'objet isolé de tout contexte, et l'image syntagmatique, c'est-à-dire celle où l'objet est situé dans un enchaînement d'objets, dans son environnement. Ainsi, l'ancre représente-t-elle une illustration paradigmatique, car en l'occurrence elle n'est jamais rattachée à un bateau ou même à une chaîne, alors que l'ambulance militaire constitue l'une des illustrations syntagmatiques des articles de notre corpus dans le dictionnaire de 1906, elle est présentée en 
effet à côté d'une tente munie du drapeau de la croix rouge, avec des soldats infirmiers affairés autour d'un blessé sur une civière. Et en restant dans le même type d'analyse, il conviendrait aussi de distinguer selon la formule de Josette Rey-Debove ${ }^{9}$ les illustrations totales, présentant une totalité, et les illustrations partielles ne donnant qu'une partie du référent à illustrer: ainsi à l'article «aigle» de l'édition de 1878 correspond une illustration partielle, seule la tête est représentée, alors qu'en 1906, l'illustration est à la fois totale, l'aigle y est en pied, et syntagmatique, il y est représenté sur son aire, avec une proie dans les serres qu'il va offrir aux aiglons.

Quant à la planche, détachée de l'article, elle est à proprement parler, le plus souvent, une illustration terminologique sur un thème introduit par une unité de la nomenclature. Mais elle peut prendre une certaine autonomie, au point même qu'elle n'est pas toujours située à sa place alphabétique par rapport à la nomenclature. Selon la définition même du Petit Larousse 1994, une planche est une «illustration ou (un) ensemble d'illustrations relatives à un même sujet et occupant dans un livre la plus grande partie ou la totalité d'une page». Il est clair qu'ici la référence est externe, mais qu'il reste parfois difficile, selon des critères internes, de dissocier une illustration complexe très fournie d'une planche réduite à une demi-page et dépourvue de commentaires annexes qui la dissocieraient nettement de l'illustration d'un article correspondant à un archilexème.

\section{Analyses comparatives et premiers constats}

Les premiers constats révélés par l'analyse du corpus choisi, la lettre A de douze éditions du «Petit Larousse», sont d'ordre quantitatif. En effet, même si nos décomptes peuvent ne pas être parfaitement exacts, à quelques unités près, les chiffres sont d'emblée très parlants. Ainsi, entre 1879 et 1993, d'une part, nous sommes passés de 74 illustrations à 119 , avec un cap à 166 en $1959(1879: 74 ; 1889: 79 ; 1906: 159 ; 1948: 155 ; 1952: 155$; $1959: 166 ; 1968: 124 ; 1973: 124 ; 1981: 113 ; 1989: 119 ; 1994: 119)$ ce qui correspond à une augmentation de $60 \%$, et, d'autre part, ce qui est encore plus significatif, par le biais des illustrations complexes et des planches, nous sommes passés de 71 termes supportés par les illustrations en 1889 (l'édition de 1878 ne comportait ni illustration complexe ni planche) à 822 en 1981 et 728 en 1994, ce qui cette fois-ci correspond à une augmentation de $1025 \%$ !

Par ailleurs, si nous relevons plus précisément dans notre corpus ce qui doit être attribué aux illustrations complexes d'une part et aux planches d'autre part, la distinction est également très révélatrice. Ainsi, au sein des articles, de par les illustrations complexes, notamment terminologiques et ordonnées qui apparaissent pour la première fois dans l'édition de 1906, on passe de 58 termes repérés en 1906 à 390 en 1994 (1906: 58; 1935 : $50 ; 1948: 45 ; 1952 ; 45 ; 1959: 245 ; 1968: 342 ; 1981: 425 ; 1989: 390 ; 1994: 394)$, soit presque sept fois plus. Quant aux planches, les premières datant de 1889 , on passe de 71 termes en 1889 à 338 en 1994 (1889: 71 avec deux planches; 1906: 212 avec 8 planches; 1935 : 231 avec dix planches; 1948: 197 avec 10 planches; $1952: 217$ avec 12 planches; 1959: 237 avec 14 planches sans compter les planches réservées à l'art et aux peintures; 1968: 445 termes dont 237 pour la seule anatomie; $1981: 397$ avec 16 planches; 1989: 338 en rajoutant l'astronomie et l'anatomie distribuée dans la lettre B; 1994: idem), soit presque cinq fois plus.

Étudiée au sein de notre corpus, l'augmentation des illustrations complexes est de fait particulièrement significative et mérite que l'on s'y attarde. Ainsi, en 1879, aucune illustration n'est légendée, toutes sont monofigurales et monosémiques: c'est le règne de l'illustration simple et de l'illustration paradigmatique. Dès 1906 apparaissent par contre les illustrations complexes avec 4 illustrations terminologiques (alambic, ancre, arçon, ascenseur), 20 illustrations typologiques (abaque, abat-jour, abat-vent, abeilles, aile, 
acrobates, décade, allonges, alpenstocks, amassettes, angle, types d'antennes, types d'anthères, francs archers, arrosoirs, as, assemblages, attelles, attisoirs, auges), une illustration à la fois typologique et ordonnée (assemblage 1-en bois, 2-en fer), une ilustration plurielle et ordonnée (ascenseur) dont une figure terminologique, et 11 illustrations syntagmatiques (accubitum, aigle et ses aiglons, aiguilles et aiguilleurs, alligator, altise, ambulance militaire, aorte, autour, appentis, auvent, avocette), ce qui correspond pour l'édition de 1906 à $22 \%$ d'illustrations complexes, en y incluant les illustrations syntagmatiques, et à peine $16 \%$ si on ne les prend pas en compte. Inversement, pour l'édition de 1994, les illustrations simples (par exemple l'agami, l'agouti présents depuis 1879, l'agneau, l'addax présents depuis 1981, l'arquebuse à mèche également présente depuis 1879 , mais après un passage par l'arquebusier de 1906 à 1989) ne représentent qu'un peu plus d'une trentaine d'illustrations, c'est-à-dire seulement $29 \%$ de l'ensemble des illustrations et les $71 \%$ qui restent sont constitués d'illustrations complexes.

Quant aux illustrations terminologiques qui représentaient seulement $12 \%$ des illustrations en 1906, en 1994 elles sont passées à $43 \%$. Au reste, s'il faut se fier aux détails révélateurs, c'est une tendance qui, d'édition en édition, se confirme, y compris de 1989 à 1994 où les illustrations sont pourtant identiques à $98 \%$. Ainsi, l'une des rares illustrations qui, en dehors de la colorisation systématique, ait été transformée, est celle représentant les actinies (plus couramment appelées les anémones de mer): en 1989, celles-ci sont illustrées de manière syntagmatique (l'une des deux actinies est en train d'absorber un poisson) sans que la figure soit terminologique, alors qu'en 1994 l'illustration n'est plus syntagmatique (ou très peu) mais devient par contre nettement terminologique, l'information y étant relayée par un microsystème lexical constitué de quatre termes (bouche, pharynx, pied adhésif, tentacule).

Enfin, en ce qui concerne les illustrations ordonnées qui apparaissent timidement en 1906 ( $1 \%$ !), elles ne cessent d'augmenter d'édition en édition pour arriver à $7 \%$ en 1994 , ce qui est considérable. En effet l'illustration ordonnée, presque toujours polyfigurale, représente un choix coûteux en espace typographique, aussi leur présence contrevenant au principe d'économie, elles ne sont choisies que lorsque le sujet les rend presque incontournables. Citons par exemple l'illustration ordonnée correspondant à l'article «accouchement» ou à la «fabrication de l'acier».

Ainsi, parmi les illustrations complexes, dans leur ensemble en constant développement, même les plus coûteuses en place n'ont cessé d'augmenter. Et, installés au cœur des illustrations complexes, se sont développés des microsystèmes lexicaux dont les unités à l'échelle du dictionnaire se comptent par milliers de mots. Afin de mieux concrétiser cette évolution, nous avons effectué hors corpus un rapide relevé des termes ainsi présentés dans la totalité de la «partie langue» pour trois éditions significatives, l'édition de 1889 , qui est la première à offrir des planches et des illustrations complexes, ensuite l'édition de 1906 qui innove de manière marquante dans le domaine des illustrations, toutes légendées, et enfin l'édition de 1994, la plus récente et où les illustrations sont toutes en couleurs. En 1889, environ 810 termes sont relevés, en 1906 nous en comptons 2800 et en 1994 nous en dénombrons 6280 . C'est une progression qui ne peut nous laisser indifférents.

\section{Des légendes motivées}

Les légendes sont l'objet d'une évolution étonnante, particulièrement sensible à travers le métalangage qu'elles abritent depuis l'édition de 1989. En effet, à la simple mention de l'animé ou de l'inanimé illustré, se substitue fréquemment depuis cette édition. une légende assortie d'un métalangage propre à expliciter la finalité poursuivie, en quelque sorte une légende motivée. 
L'analyse systématique de l'ensemble des illustrations de toute la «partie langue» des éditions de 1989 et de 1994, au-delà donc de la lettre A, nous a permis de classer les différents syntagmes utilisés dans les légendes, en fonction des objectifs poursuivis.

- Il faut en effet relever tout d'abord ceux qui servent à distinguer une partie du référent ou des référents possibles, le plus souvent introduisant des illustrations typologiques ou partielles. Ainsi en est-il des formules suivantes:

- «Exemple de...» («mosaïque»/《d'ornements»/《de lichen»/ «d'illusions optiques»/ «de prothèse interne»/《de voiture hippomobile»/etc.; ou encore «météorologie : exemple de carte de situation»/ / fortification : exemple d'ouvrage fortifié du XVIII ${ }^{\mathrm{e}}$ siècle»/etc.).

- «Détail de...» («de l'œil»/《du vitrail»/«d'une plume d'oie»/etc.; ou encore «galvanomètre à cadre mobile : détail»/《ordre, détail d'une colonne»/etc.).

- «Différents...» («différentes pièces constituant une charpente de bois»/ «différentes opérations de filature de coton»/etc.; ou encore «échelle des plans : différents cadrages»/ «aéroport : différentes conceptions»/etc.).

- «Quelques... («quelques volumes caractéristiques»/《quelques opérations de tournage»/ etc.; ou encore «becs: quelques formes de bec et leur fonction»/«corne de quelques animaux» / etc.).

- «Quelques, divers, etc., types de...» («quelques types de parquets»/ «quelques types de ponts»/ «divers types de lampes»/etc.; ou encore «inflorescence : principaux types»/ «réacteurs : deux types de réacteurs nucléaires»/etc.).

- «Partie de...» («partie centrale d'une iconostase» ou encore pour «crocodiliens», «parties antérieures vues de dessus»)

- Dans le même souci de signaler l'aspect partiel de ce qui est représenté, il est intéressant de relever aussi ce qui correspond entre autres aux illustrations ordonnées avec notamment «phases d'une mitose» ou «pain: principales phases de fabrication» ou encore ce qui pourrait être considéré comme la forme elliptique d'une illustration ordonnée : "phase d'un parcours de golf».

- Se distinguent également les légendes annonçant les illustrations terminologiques: ainsi en est-il de «bœuf: désignation des morceaux de boucherie» ou de «mouton», «porc», «veau», traité de la même manière, ou encore de «arbre: dénomination des différentes parties». Se rattachant à cette catégorie, il faut évoquer par ailleurs pour «chaussure», «escalier», «planeur», «porte», etc., le rajout du syntagme nominal derrière les deux points: «éléments constitutifs». Sans oublier, pour «maison», la très explicite «nomenclature des parties ou éléments pouvant constituer une maison individuelle».

- Signalons aussi les syntagmes destinés à expliquer un fonctionnement et caractérisant des illustrations le plus souvent ordonnées. Et donc par exemple: «principe du pendule de torsion», «principe de la télécopie», «principe du télex» ou bien «ramasseusepresse : principe de fonctionnement», ou bien encore «radar», «phare», «embrayage d'automobile» suivis de «principe de fonctionnement». De la même manière, un assez grand nombre de syntagmes sont construits avec «schéma» et «schématique»: «schéma du traitement de l'eau», «schéma de principe de la fusion thermonucléaire», ou bien encore «schéma d'élaboration» introduit par deux points derrière «acier», «aluminium», «caoutchouc». De même que l'on repère dans la planche consacrée à l'imagerie médicale le «schéma du fonctionnement, d'un appareil d'échographie», ou pour le "propfan», les «microscopes» et la «montre», le «schéma de principe». Auxquels se rajoutent la «vue schématique de la galaxie», et pour le téléphone, la «représentation schématique d'un réseau».

- Il importe d'introduire maintenant une dernière catégorie: celle qui fait explicitement appel au métalangage du graphisme. En l'occurrence, au-delà de la «vue schématique», de la «vue d'ensemble» (pour «route», «mosquée», «télévision», etc.), de la «vue 
en coupe» («oreille»), de la «vue antérieure» et «postérieure» («estomac», «jambe», etc.), de la «vue supérieure» («œil»), «de dessus» («rémora»), «de dessous» (la «patte» du chat), «de face» et «de dos» («tétras-lyres»), «extérieure» («larynx»), «externe» et «interne» (escargot), «de profil» («sabot de cheval»), il faut surtout évoquer le terme très technique d'《écorché» avec, entre autres, l'«écorché d'une berline 205 Peugeot» ou pour le «cornsheller», l'«écorché montrant le fonctionnement», ou bien encore pour «métro: écorché d'une rame MF 77 utilisée par la R.A.T.P. à Paris» ainsi que pour «moissonneusebatteuse : écorché et schéma de fonctionnement».

Pour conclure ces quelques propos consacrés à la métamorphose des légendes, une métamorphose constatée à partir de l'édition de 1989, il faut signaler, même s'il ne s'agit plus ici de technolectes, que le souci marqué et croisssant de précision dans la légende se révèle aussi particulièrement visible pour toutes les illustrations consacrées aux animaux. Ainsi, le «têtard»y devient le «têtard de la grenouille», le «cygne», le «cygne muet de Sibérie», le «taureau», le «taureau de race frisonne», le «hibou», le «hibou moyen duc», etc. Le procédé est sytématique et il reflète bien cette volonté des auteurs de faire de l'illustration une espèce du genre défini par l'article, un exemple de l'article considéré comme générique, une réalisation du concept qui y est défini, une illustration possible d'une totalité par définition insaisissable et infinie. En quelque sorte une «citation visuelle» dont on précise bien la nature et l'origine...

Vaste champ d'étude en définitive que celui à ouvrir sur les illustrations dictionnairiques. Délicate relation également à étudier que celle qui s'établit entre les illustrations et les technolectes, au sein d'un dictionnaire. Curieux paradoxe enfin que celui qui consiste à retrouver l'onomasiologie, par le biais des illustrations, dans le corps même d'un dictionnaire sémasiologique. Car l'illustration complexe, notamment terminologique, devient bel et bien le relais efficace de milliers de mots ainsi regroupés en microsystèmes lexicaux.

Ainsi, mieux connaître, mieux comprendre et à terme offrir une typologie et une analyse pertinentes des mécanismes dictionnairiques en jeu, tels pourraient être les objectifs des recherches qui s'installeraient dans ce secteur dictionnairique. Un secteur qui, il faut bien le reconnaître, est assez mal connu et dans lequel les dictionnaristes eux-mêmes paraissent agir encore intuitivement.

Si un tel objectif était atteint, puissent alors ces recherches s'inscrire dans les perspectives tracées par Bernard Quemada au seuil de son étude incomparable et lumineuse, Les dictionnaires du français moderne. 1539-1863: «Notre propos essentiel, y annonce-il en effet, est d'apporter une contribution concrète et pratique à la connaissance encore trop approximative des réalisations lexicographiques françaises.» Voilà un programme très clair. Et un modèle extraordinairement galvanisant pour tous ceux qui se lanceraient dans ce type de recherche.

Notes

1. Guilbert, Louis (1973) : «La spécificité du terme scientifique et technique», Langue française, $\mathrm{n}^{\circ} 17$, p. 10.

2. Rey, Alain (1992: 46).

3. Cusin-Berche, Fabienne et Michèle Perret (1992): «Présentation», «Les français professionnels», Linx, $n^{\circ} 27$, p. 7.

4. Rey-Debove, Josette $(1971: 34)$.

5. Issues du Petit Larousse de 1959: abricot (photographie inversée), accotement, accumulateur, agami, agouti (inversé), aigle (l'illustration correspondant au rapace), aigle (l'emblème), aine, aisselle, albatros, alouette, alpaga, amandier, amazone, amygdales, anche, anémone, ânes, angles (quelques-uns), anguille, antilope, aorte, appendice, ara, araignées, arc, arc-boutant, arçon, armes et armoiries, arnica, artichaut, aspic, aubépine, aubergine, autruche, avocat, avoine, azimut.

6. Issues du Nouveau Petit Larousse de 1968: abdomen, abeille, acacia, accord, actinie, angles adjacents, ail, aile, airelle, ajonc, alambic, amble, amortisseur, anchois, ancres, arachide, arbre, armure, arpège, asperge.

7. À titre indicatif, voici les 28 mots-centres illustrés par une planche : architecte $(1$ page $)$, armée $(1 \mathrm{p}$.), automobile (1 p.), avion (1 p.), bateau (2 p.), botanique (1 p.), charpente (1 p.), chemin de fer (1 p.), cheval 
(1 p.), chimie (1 p.), corps humain ( 2 p.), église-cathédrale (1 p.), escrime (1 p.), acier (1 p.), fortification (1 p.), héraldique $(1 / 2$ p.), imprimerie (1 p.), insecte $(2 / 3$ p.), mammifere $(1 / 2$ p.), maison (1 p.), mine (1 p.), moteur $(1 \mathrm{p}$.$) , nœuds ( 2 \mathrm{p}$. incomplètes $)$, pétrole $(1 \mathrm{p}$.$) , radiodiffusion (1 / 3 \mathrm{p})$, serrure $(1 \mathrm{p}$.$) , télévision$ (1 p.), tissage ( $1 / 4$ p.).

À ceux-là s'ajoutent 10 illustrations constituées d'une seule figure concernant les mots-centres : astrologie, cerf, chien, cloche, couronne, croix, échecs, harnais, orgue, tissu.

8. Cette expression apparaît indirectement pour la première fois dans la préface de l'édition de 1952 où sont évoquées «la partie consacrée à la langue française» qui se «complète désormais d'un supplément grammatical» ainsi que la «partie Arts, Lettres, Sciences» dont la nomenclature est renouvelée. En 1959, cette dénomination devient effective dans la préface où la «partie langue» et la «partie Arts, Lettres, Sciences» sont directement citées. De même que, parallèlement dans cette édition, les Tableaux et principales illustrations en noir sont scindés en fonction de ces deux parties reprises en sous-titre.

Ajoutons ici que dans notre analyse rapide des éditions se succédant de 1879 à 1994, tout ce qui est cité est tiré des préfaces. Celles-ci sont adressées à partir de 1906 «Aux lecteurs» et ne dépassent jamais deux pages, à l'exception de celle rédigée par Pierre Larousse pour l'ouvrage initial de 1856, Préface de huit pages reprise dans l'édition de 1879 et précédant la Préface propre à cette édition.

9. Rey-Debove, Josette $(1971: 35)$.

\section{RÉFÉRENCES}

Langue française (février 1973): $\mathrm{n}^{\circ} 17$, «Les Vocabulaires techniques et scientifiques», numéro dirigé par $\mathrm{L}$. Guilbert et J. Peytard, Paris, Larousse.

Linx (1992) : $n^{\circ}$ 27, «Les Français Professionnels», numéro préparé par M. Perret et F. Cusin-Berche, Nanterre, Centre de Recherches Linguistiques Paris X.

PRUVOST, Jean (1983) : «Le dictionnaire analogique: Boissière et ses successeurs», Le français moderne, 51, pp. 193-204.

QUEMADA, Bernard (1967): Les dictionnaires du français moderne. 1539-1863, Étude sur leur histoire, leurs types et leurs méthodes, coll. «Études lexicologiques», $\mathrm{n}^{\circ} 1$, Paris, Didier.

REY, Alain (1992) : La terminologie, noms et notions, coll. «Que sais-je ?», $\mathrm{n}^{\circ} 780$, deuxième édition corrigée, Paris, PUF.

REY-DEBOVE, Josette (1971): Étude linguistique et sémiotique des dictionnaires français contemporains, coll. «Approaches to Semiotics», $n^{\circ} 13$, The Hague / Paris, Mouton. 